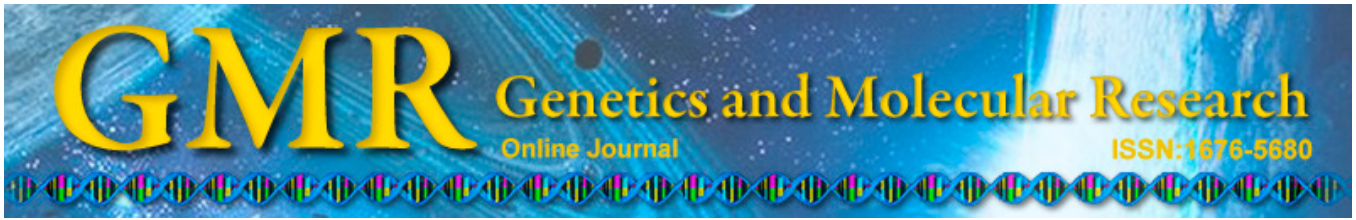

\title{
Cyclin D1 G870A gene polymorphism and risk of leukemia and hepatocellular carcinoma: a meta-analysis
}

\author{
Y. Zhao ${ }^{1,2 *}$, H.R. He ${ }^{1 *}$, M.Y. Wang ${ }^{1}$, X.D. Ren ${ }^{1}$, L. Zhang1, Y.L. Dong ${ }^{1}$ \\ and J. Lu ${ }^{1}$ \\ ${ }^{1}$ Department of Pharmacy, The First Affiliated Hospital of Medical College, \\ Xi'an Jiaotong University, Xi'an, China \\ ${ }^{2}$ College of Pharmacy, Xi'an Medical University, Xi'an, China \\ *These authors contributed equally to this study. \\ Corresponding author: J. Lu \\ E-mail: lujun2006@mail.xjtu.edu.cn
}

Genet. Mol. Res. 14 (2): 5171-5180 (2015)

Received May 13, 2014

Accepted November 24, 2014

Published May 18, 2015

DOI http://dx.doi.org/10.4238/2015.May.18.7

\begin{abstract}
Cyclin D1 (CCND1) is a key protein involved in cellcycle regulation, and the CCND1 G870A polymorphism is associated with many types of malignancy. Studies examining the associations between this G870A polymorphism and susceptibility to leukemia and hepatocellular carcinoma (HCC) have shown inconsistent results. Therefore, we conducted a meta-analysis to clarify these associations. A search of the PubMed database yielded 7 relevant articles: 3 pertaining to leukemia and 4 to HCC. The odds ratios (ORs) from individual studies were pooled using a fixed or random-effect model. A significant association was observed between the CCND1 G870A variant and leukemia under the allele contrast model $[\mathrm{P}=0.003, \mathrm{OR}=1.49,95 \%$ confidence interval $(\mathrm{CI})=1.15-1.95]$, the homozygote contrast model $(\mathrm{P}=0.003, \mathrm{OR}=2.30,95 \% \mathrm{CI}=1.34-3.96)$, and the recessive model $(\mathrm{P}$ $=0.002, \mathrm{OR}=2.03,95 \% \mathrm{CI}=1.29-3.21)$. A significant association was observed between this variant and HCC under the recessive model $(\mathrm{P}=$
\end{abstract}


$0.0006, \mathrm{OR}=1.62,95 \% \mathrm{CI}=1.23-2.14)$, the dominant model $(\mathrm{P}=0.002$, $\mathrm{OR}=1.59,95 \% \mathrm{CI}=1.19-2.14)$, the homozygote contrast model $(\mathrm{P}<$ $0.0001, \mathrm{OR}=2.06,95 \% \mathrm{CI}=1.45-2.94)$, and the allele contrast model $(\mathrm{P}<0.0001, \mathrm{OR}=1.43,95 \% \mathrm{CI}=1.20-1.69)$. Our findings suggest that heritable CCND1 status may influence the risk of developing leukemia and HCC, and that more attention should be given to carriers of these susceptibility genes.

Key words: Cyclin D1 G870A; Hepatocellular carcinoma; Leukemia; Polymorphism; Susceptibility

\section{INTRODUCTION}

Cancer is a type of cell-cycle disease (Clurman and Roberts, 1995), and tumors and tumor cell lines have been examined in many studies for the presence of mutations in genes encoding cell-cycle-related proteins (Clurman and Roberts, 1995; Funk and Kind, 1997). Some important cell-cycle regulators may be directly involved in oncogenesis (Hunter and Pines, 1991, 1994).

Cyclins are essential to cell-cycle regulation, as evidenced by the specific and periodic expressions of members of this protein family, such as cyclins $\mathrm{A}, \mathrm{B}$, and $\mathrm{D}$, during cell-cycle progression (Evans et al., 1983). Cyclin D1, whose encoding gene (CCND1) is located on human chromosome 11q13, is a key regulatory protein at the G1/S checkpoint of the cell cycle (Hu et al., 2014). The guanine/adenine polymorphism identified at nucleotide 870 [National Center for Biotechnology Information single-nucleotide polymorphism (SNP) cluster ID: rs603965], codon 242 in exon 4 of the CCND1 gene (Betticher et al., 1995), has recently received considerable attention after it was discovered that the A allele may interfere with splicing to generate a variant splice product, transcript $b(\mathrm{Lu}$ et al., 2003; Knudsen et al., 2006; Liu et al., 2012). The protein encoded by transcript $\mathrm{b}$ (A allele) has a longer half-life than that encoded by transcript a (G allele) (Lu et al., 2003; Solomon et al., 2003; Liu et al., 2012). This strongly suggests that individuals with a higher number of copies of the CCND1 G870A polymorphism are more likely to bypass the G1/S checkpoint, thus contributing to cancer development (Lu et al., 2003; Solomon et al., 2003; Liu et al., 2012).

The relationships between the G870A SNP and excessive CCND1 activity, as well as numerous human tumors, have been studied in recent years, including primary brain tumors, bladder cancer, colorectal cancer, esophageal cancer, hepatocellular carcinoma, leukemia, and breast cancer (Akkiz et al., 2010; Zhuo et al., 2012; Bedewy et al., 2013; Sameer et al., 2013; Zeybek et al., 2013; Li et al., 2014). Among these, the numerous relevant epidemiologic studies investigating the relationship between the CCND1 G870A variant and hepatocellular carcinoma (Zhang et al., 2002; Pakakasama et al., 2004; Akkiz et al., 2010; Hu et al., 2014) or leukemia (Howe and Lynas, 2001; Hou et al., 2005; Bedewy et al. 2013) have yielded conflicting and controversial results. There has been no systematic or quantitative assessment of the published findings on this topic. Therefore, a meta-analysis of case-control studies was conducted to summarize the available evidence on CCND1 G870A in hepatocellular carcinoma and leukemia to derive a more precise estimation of the relationship between the polymorphism in these 2 diseases. 


\section{MATERIAL AND METHODS}

\section{Search strategy}

We carried out a comprehensive search of relevant published studies from database initiation until May 14, 2014 using the MEDLINE (PubMed) database. The search strategy was based on a combination of the following keywords: "G870A", "CCND1 (cyclin D1)", "leukemia", "liver cancer" ("hepatocellular carcinoma" or "HCC"), "polymorphism", "susceptibility", and "risk". Only journal articles were included in the analysis and the language was limited to English. All references cited in the studies were reviewed to identify additional relevant study.

\section{Study selection}

Published studies were included if they: i) used a case-control design; ii) evaluated the association between the G870A polymorphism and susceptibility to leukemia or hepatocellular carcinoma; and iii) presented sufficient data on the distribution of CCND1 gene polymorphism in cases and in control groups, or data necessary to calculate these. When multiple publications from the same study were available, we used the publication with the largest number of cases and most applicable information.

\section{Data extraction}

For each eligible study, 2 investigators independently carried out the eligibility evaluation and data abstraction. Any disagreements were further discussed and resolved by consensus. Data abstracted from each study included: name of the first author, year of publication, ethnicities of the study population, and genotype distributions of the cases and controls. We also tested the distribution of the genotypes in controls for departure from Hardy-Weinberg equilibrium (HWE). The study was excluded from meta-analysis if the distribution of the genotypes in controls were not accordance with HWE.

\section{Statistical analysis}

The meta-analysis examined the overall association between the allele contrasts (A vs $\mathrm{G})$, the contrast of homozygotes (AA vs GG), the recessive models (AA vs $\mathrm{AG}+\mathrm{GG}$ ), and the dominant models (AA+AG vs GG).

The raw data for genotype distribution were used to calculate study-specific estimates of odds ratios (ORs) and 95\% confidence intervals (CIs). The Review Manager (version 5.2) software was used for the meta-analysis.

Heterogeneity between studies was tested using the Q statistic. Such heterogeneity was considered statistically significant if $\mathrm{P}<0.10$. Heterogeneity was quantified using the $\mathrm{I}^{2}$ metric, which is independent of the number of studies in the meta-analysis $\left(\mathrm{I}^{2}<25 \%\right.$ no heterogeneity; $\mathrm{I}^{2}=25-50 \%$ moderate heterogeneity; $\mathrm{I}^{2}>50 \%$ large or extreme heterogeneity) (Wang et al., 2010).

The overall pooled OR and 95\% CI were estimated using the Mantel-Haenszel method with a fixed-effect model when no significant clinical or statistical heterogeneity was pres- 
ent ( $P \geq 0.10)$ (Gurion et al., 2013). When substantial heterogeneity was present, the randomeffect model was used. The significance of the pooled OR was determined using the $Z$ test. The level of statistical significance was set at $\mathrm{P}<0.05$.

Potential publication bias was estimated by constructing funnel plots. If most of the data appeared at the top of a funnel plot and was distributed roughly symmetrically, the absence of obvious publication bias and vice versa were suggested (Egger et al., 1997). There was no need to construct funnel plots when there were too few analyzed studies.

\section{RESULTS}

\section{Search of published reports}

A flow chart depicting the study selection process is shown in Figure 1. A total of 1737 articles were selected based on various combinations of the keywords listed in the Material and Methods section. Evaluation for duplicates resulted in the removal of 522 articles. Of the remaining 1215 articles, 705 did not examine the relationship between the CCND1 polymorphism and leukemia or hepatocellular carcinoma or only studied the effect of drugs, $400 \mathrm{did}$ not report an association between the CCND1 gene G870A polymorphism and susceptibility to leukemia or hepatocellular carcinoma, 84 articles mainly focused on other diseases, and 17 were review articles. After excluding these articles, only 9 studies examining the relationship between CCND1 gene G870A polymorphism and the risk of leukemia or hepatocellular carcinoma remained. However, 2 of these did not provide sufficient data and were also excluded. Therefore, only 7 studies qualified for inclusion in this meta-analysis, among which 3 focused on leukemia and 4 examined hepatocellular carcinoma.

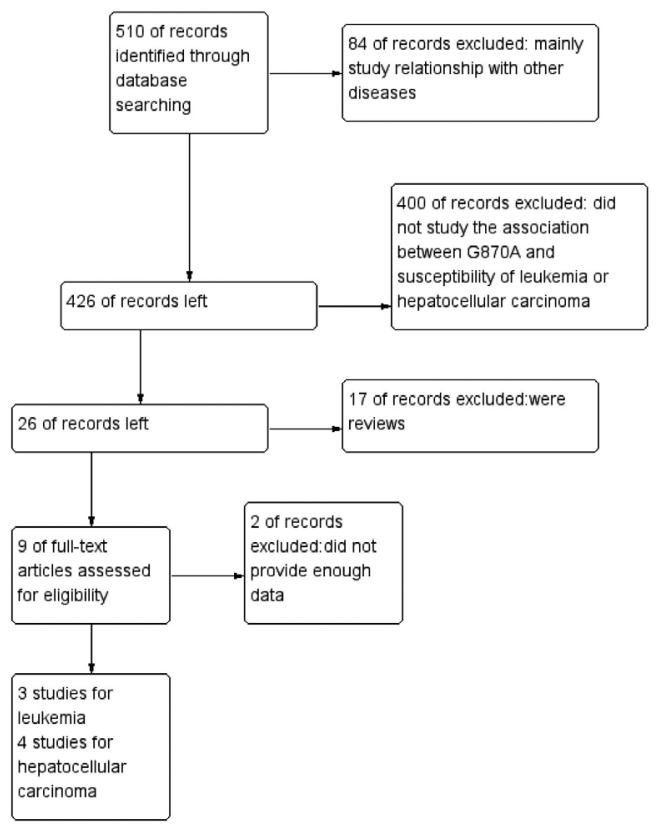

Figure 1. Flow chart of study selection. 


\section{Study characteristics}

Characteristics of the 7 articles included are listed in Table 1. In the 3 studies examining leukemia, containing 227 cases and 218 controls, the distribution of the G870A genotypes in controls were in accordance with HWE. In the study by Pakakasama et al. (2004), which described the association between the G870A SNP and hepatocellular carcinoma risk in 3 races, the distribution of the G870A genotypes in controls of Hispanics were not in accordance with HWE and this study was therefore excluded from the meta-analysis. The study by Pakakasama et al. (2004) examining African Americans and Caucasians and 3 other studies (Zhang et al., 2002; Akkiz et al., 2010; Hu et al., 2014), containing 540 cases and 626 controls, were included in the meta-analysis to determine the role of the G870A polymorphism on hepatocellular carcinoma risk.

\begin{tabular}{|c|c|c|c|c|c|c|c|c|c|}
\hline \multirow[t]{2}{*}{ Study } & \multirow[t]{2}{*}{ Year } & \multirow[t]{2}{*}{ Ethnicity } & \multicolumn{3}{|c|}{ Genotype distribution in cases } & \multicolumn{3}{|c|}{ Genotype distribution in controls } & \multirow[t]{2}{*}{$\mathrm{P}_{\mathrm{HWE}}$ in controls } \\
\hline & & & $\overline{\mathrm{GG}}$ & $\mathrm{AG}$ & $\overline{\mathrm{AA}}$ & GG & $\mathrm{AG}$ & $\mathrm{AA}$ & \\
\hline \multicolumn{10}{|l|}{ Leukemia } \\
\hline Howe et al. & 2001 & British & 7 & 9 & 3 & 4 & 7 & 2 & 0.710 \\
\hline Hou et al. & 2005 & Chinese & 41 & 88 & 54 & 55 & 101 & 34 & 0.293 \\
\hline Bedewy et al. & 2013 & Egyptians & 4 & 12 & 9 & 7 & 7 & 1 & 0.667 \\
\hline \multicolumn{10}{|l|}{$\begin{array}{l}\text { Hepatocellular } \\
\text { carcinoma }\end{array}$} \\
\hline Zhang et al. & 2002 & Taiwanese & 9 & 50 & 38 & 3 & 19 & 13 & 0.281 \\
\hline \multirow[t]{3}{*}{ Pakakasama et al. } & 2004 & Caucasians & 17 & 25 & 15 & 56 & 71 & 32 & 0.278 \\
\hline & & African Americans & 2 & 3 & 1 & 33 & 16 & 3 & 0.576 \\
\hline & & Hispanics & 7 & 4 & 4 & 28 & 17 & 17 & 0.0006 \\
\hline Akkiz et al. & 2010 & Turkish & 38 & 69 & 53 & 56 & 73 & 31 & 0.413 \\
\hline Hu et al. & 2014 & Chinese & 32 & 119 & 69 & 45 & 124 & 51 & 0.057 \\
\hline
\end{tabular}

\section{Meta-analysis results}

Figure 2 shows the main results of this meta-analysis regarding the association between the CCND1 G870A polymorphism and the susceptibility to leukemia. This SNP was associated with leukemia susceptibility under the recessive model $(\mathrm{P}=0.002, \mathrm{OR}=2.03,95 \% \mathrm{CI}$ $=1.29-3.21$, Figure 2A). However, no association was found under the dominant model (Figure 2B). In addition, significant associations were observed under the contrast of homozygotes $(\mathrm{P}=0.003, \mathrm{OR}=2.30,95 \% \mathrm{CI}=1.34-3.96$, Figure $2 \mathrm{C})$ and the allele contrast model $(\mathrm{P}=$ $0.003, \mathrm{OR}=1.49,95 \% \mathrm{CI}=1.15-1.95$, Figure $2 \mathrm{D}$ ).

Figure 3 shows the main results of this meta-analysis regarding the association between the CCND1 G870A polymorphism and the susceptibility to hepatocellular carcinoma. In general, we found that the G870A polymorphism was significantly associated with hepatocellular carcinoma risk under the recessive model $(\mathrm{P}=0.0006, \mathrm{OR}=1.62,95 \% \mathrm{CI}=1.23-2.14$, Figure $3 \mathrm{~A})$, dominant model $(\mathrm{P}=0.002, \mathrm{OR}=1.59,95 \% \mathrm{CI}=1.19-2.14$, Figure $3 \mathrm{~B})$, homozygotes contrasting $(\mathrm{P}<0.0001, \mathrm{OR}=2.06,95 \% \mathrm{CI}=1.45-2.94$, Figure $3 \mathrm{C})$, and allele contrast model $(\mathrm{P}<0.0001, \mathrm{OR}=1.43,95 \% \mathrm{CI}=1.20-1.69$, Figure $3 \mathrm{D})$. 


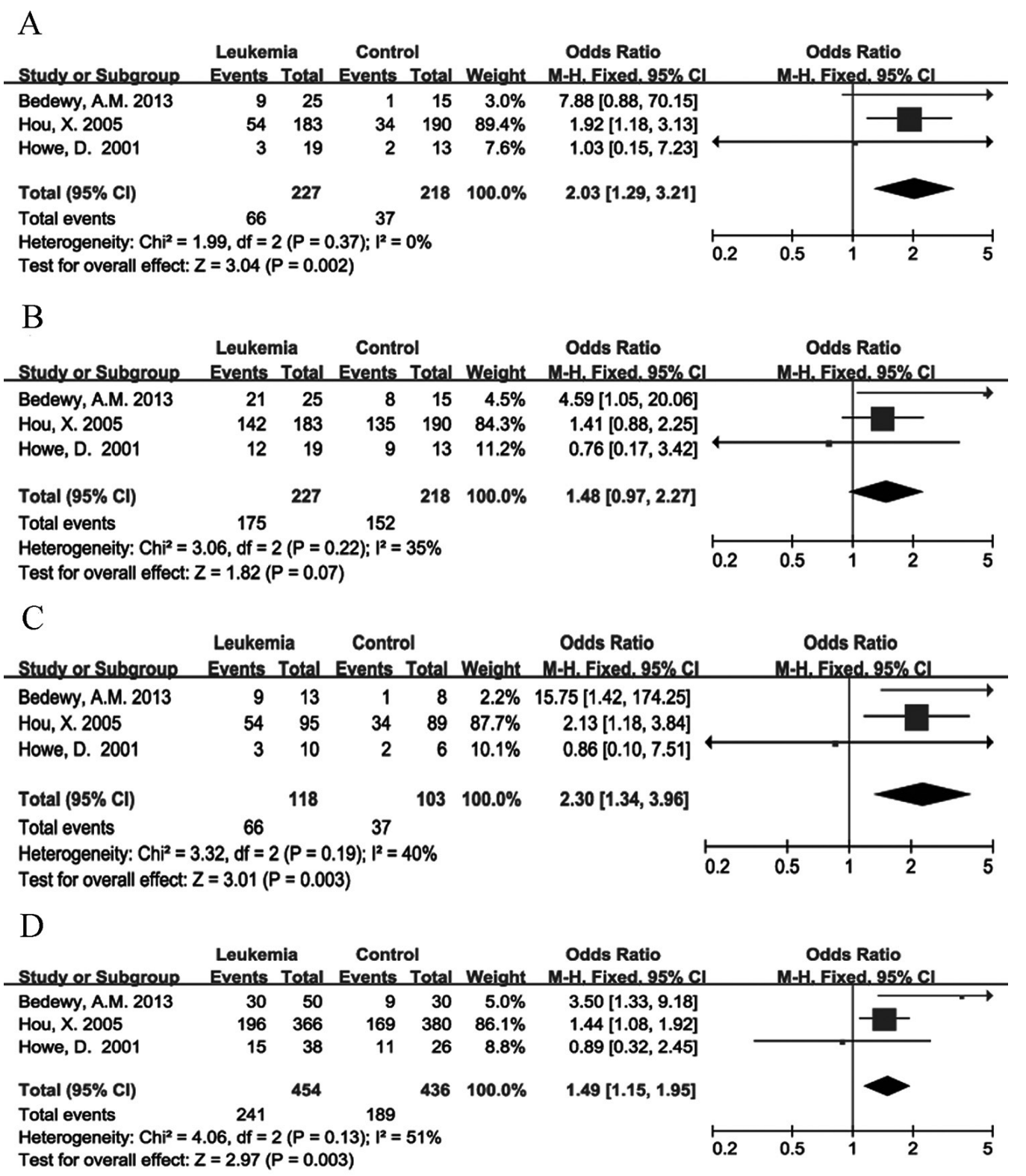

Figure 2. A. Meta-analysis of the association between CCND1 G870A variant and leukemia under the recessive model. B. Meta-analysis of the association between CCND1 G870A variant and leukemia under the dominant model. C. Meta-analysis of the association between CCND1 G870A variant and leukemia under homozygotes contrasting. D. Meta-analysis of the association between CCND1 G870A variant and leukemia under allele contrasting. 


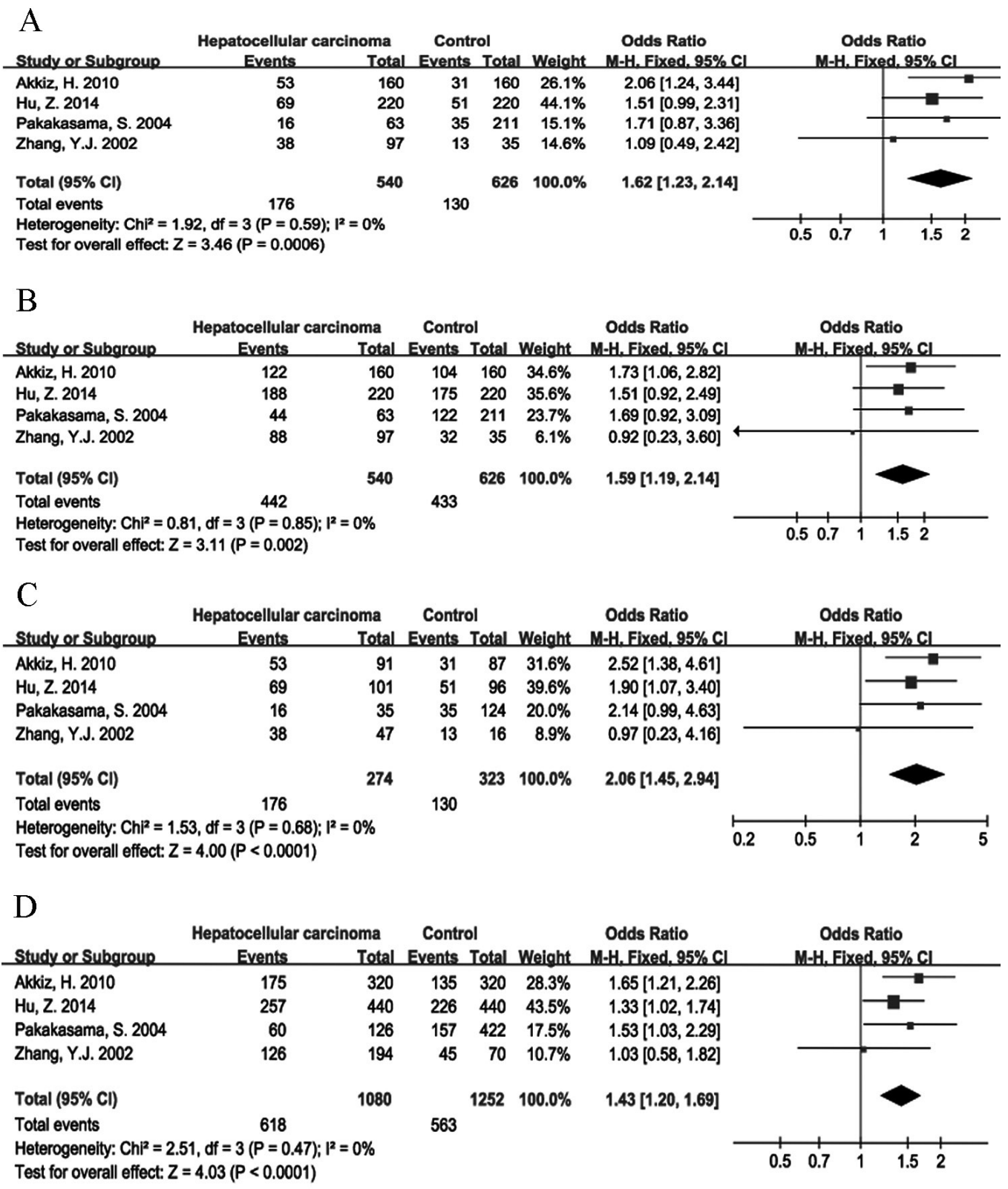

Figure 3. A. Meta-analysis of the association between CCND1 G870A variant and hepatocellular carcinoma under the recessive model. B. Meta-analysis of the association between CCND1 G870A variant and hepatocellular carcinoma under the dominant model. C. Meta-analysis of the association between CCND1 G870A variant and hepatocellular carcinoma under homozygotes contrasting. D. Meta-analysis of the association between CCND1 G870A variant and hepatocellular carcinoma under allele contrasting. 


\section{DISCUSSION}

The possible association between the CCND1 G870A polymorphism and susceptibility to leukemia or hepatocellular carcinoma has been examined in many recent studies, both domestic and overseas. Our selection criteria yielded 7 published articles, including 3 examining the CCND1 G870A polymorphism and susceptibility to leukemia and 4 examining the CCND1 G870A polymorphism and susceptibility to hepatocellular carcinoma. These articles described studies that employed reasonably robust methods that yielded sufficient data and definitive results. The results of the present meta-analysis showed that either the AA genotype or the A allele increased the risk of leukemia, and that the CCND1 G870A SNP increased the risk of hepatocellular carcinoma under the recessive, dominant, homozygotes contrast, and allele contrast models.

The findings of the various studies regarding the potential association between the CCND1 G870A SNP and the risk of leukemia or hepatocellular carcinoma have been inconsistent. Thus, a systematic evaluation method to rationalize the findings regarding these types of cancer is needed. Meta-analysis is recognized as one of the best methods for secondary analysis (Sacks et al., 1987), and was therefore used in the present study to systematically summarize and analyze the relevant identified literature. During the analysis process, an analytical method was implemented to optimize the results of this study. In addition, the recessive, dominant, homozygote contrast, and allele models were used to comprehensively analyze the association between the CCND1 G870A SNP and the susceptibility to the 2 malignancies.

The studies included examining the association between the CCND1 G870A SNP and susceptibility to leukemia led to different conclusions. Hou et al. (2005) found that this SNP increased the risk of acute lymphoblastic leukemia under the recessive model, and Bedewy et al. (2013) reported that the AA genotype significantly increased the risk of acute lymphoblastic leukemia compared with the GG genotype. The authors of the latter study also found that the CCND1 G870A polymorphism did not influence the susceptibility to mantle-cell lymphoma and chronic lymphocytic leukemia (Howe and Lynas, 2001). Howe and Lynas (2001) studied the distribution of the CCND1 G870A polymorphism among patients with mantlecell lymphoma and chronic lymphocytic leukemia separately. However, data for mantle-cell lymphoma were excluded from the present meta-analysis because it does not form leukemia; therefore, the data for chronic lymphocytic leukemia (Howe and Lynas, 2001) and the 2 studies (encompassing 227 cases and 218 controls) were pooled and subjected to meta-analysis. The results revealed that both the A allele and the AA genotype were risk factors for leukemia.

Conflicting findings were also reported for the included studies that investigated the association between the CCND1 G870A gene polymorphism and susceptibility to hepatocellular carcinoma. Akkiz et al. (2010) found that this polymorphism was associated with the risk of hepatocellular carcinoma, whereas Pakakasama et al. (2004) and Hu et al. (2014) suggested that this polymorphism did not affect hepatocellular carcinoma susceptibility. Hu et al. (2014) found that the CCND1 genotype distribution did not differ significantly between hepatocellular carcinoma patients and healthy controls, but their stratification analysis according to hepatitis B virus carrier status revealed that the variant genotypes containing the A allele were associated with a significantly increased risk of this malignancy among those positive for hepatitis B surface antigen. A meta-analysis based on the data from these studies revealed that the CCND1 G870A polymorphism was a risk factor under the recessive, dominant, homozygotes contrast, and allele contrast models. Importantly, Pakakasama et al. (2004) studied the distribution of the CCND1 G870A polymorphism in different races (Caucasians, African 
Americans, and Hispanics), but the G870A genotypes in the Hispanic control group were not consistent with HWE, so the study was excluded from the present meta-analysis; thus, only the data for the other 2 races were included.

The conflicting findings in the studies included may be attributable to various factors, including differences in sample size, race, age distribution, gender, and genetic and lifestyle factors. For example, Pakakasama et al. (2004) confirmed that the A/A genotype was associated with an earlier age of onset compared to the G/A or G/G genotype, and found no overall difference in genotype frequency between their cases and normal controls. They also found a discrepancy in the A allele frequency in children of different races: $43 \%$ in Caucasians, $21 \%$ in African Americans, and $41 \%$ in Hispanics. Furthermore, infection with hepatitis B virus and/or hepatitis $\mathrm{C}$ virus, cirrhosis, male gender, concurrent alcohol use, aflatoxin B1 intake, and multiple based variants (Nissen and Martin, 2002; Chen and Chen, 2002; Das et al., 2009) were found to be risk factors for hepatocellular carcinoma in their study.

Some problems were encountered during the data-integration process. First, the numbers of studies or cases included in some of the meta-analyses was low, as was the number of cases. Sample size plays an important role in predicting the association between genotypes and cancer risk in case-control studies. Therefore, the inclusion of studies with very small samples may lead to overestimation of the true association (Das et al., 2009). The results of meta-analyses that are based on relatively small numbers of studies should be interpreted with caution. Furthermore, there was some heterogeneity between several of the studies as a result of uncontrolled confounding factors and internal selection bias. Heterogeneity cannot be avoided, and a meta-analysis cannot solve problems related to confounding factors that may be inherent in the included studies. In theory, the best way to overcome the limitation of heterogeneity is subgroup or sensitivity analysis. However, it was not possible to conduct subgroup analyses in the present study because of the small number of included studies, and sensitivity analysis would have reduced the number of cases and controls, thus reducing the statistical power. Therefore, neither subgroup nor sensitivity analyses were performed. Instead, the random-effect model was used to consolidate the highly heterogeneous results by combining the clinical and methodological differences; this approach considered the heterogeneity between the various studies in full, presumably giving more conservative and more accurate results.

Three limitations of this meta-analysis should be considered when interpreting the findings. First, the conclusions are based on relatively few cases and controls, and so the statistical power was not high. Second, the results are based on unadjusted estimates; a more precise analysis should be conducted using data from individuals, allowing researchers to adjust for covariates including age, ethnicity, family history, environmental factors, and lifestyle. Finally, only published studies were included in this meta-analysis. There is always a certain degree of publication bias, and non-significant or negative findings may go unpublished.

In conclusion, the present meta-analysis revealed that the CCND1 G870A polymorphism may be a risk factor for both leukemia and hepatocellular carcinoma. Further large-scale studies examining this locus are recommended in order to confirm the findings of this study.

\section{ACKNOWLEDGMENTS}

Research supported by the National Science Foundation for Post-doctoral Scientists of China (\#20100481342; \#2013M532122) and the Fundamental Research Funds for the Central Universities of China (\#08143047). 


\section{REFERENCES}

Akkiz H, Bayram S, Bekar A, Akgollu E, et al. (2010). Cyclin D1 G870A polymorphism is associated with an increased risk of hepatocellular carcinoma in the Turkish population: case-control study. Cancer Epidemiol. 34: 298-302.

Bedewy AM, Mostafa MH, Saad AA, El-Maghraby SM, et al. (2013). Association of cyclin D1 A870G polymorphism with two malignancies: acute lymphoblastic leukemia and breast cancer. J. BUON 18: 227-238.

Betticher DC, Thatcher N, Altermatt HJ, Hoban P, et al. (1995). Alternate splicing produces a novel cyclin D1 transcript. Oncogene 11: 1005-1011.

Chen CJ and Chen DS (2002). Interaction of hepatitis B virus, chemical carcinogen, and genetic susceptibility: multistage hepatocarcinogenesis with multifactorial etiology. Hepatology 36: 1046-1049.

Clurman BE and Roberts JM (1995). Cell cycle and cancer. J. Natl. Cancer Inst. 87: 1499-1501.

Das P, Shaik AP and Bammidi VK (2009). Meta-analysis study of glutathione-S-transferases (GSTM1, GSTP1, and GSTT1) gene polymorphisms and risk of acute myeloid leukemia. Leuk. Lymphoma 50: 1345-1351.

Egger M, Davey Smith G, Schneider M and Minder C (1997). Bias in meta-analysis detected by a simple, graphical test. BMJ 315: 629-634.

Evans T, Rosenthal ET, Youngblom J, Distel D, et al. (1983). Cyclin: a protein specified by maternal mRNA in sea urchin eggs that is destroyed at each cleavage division. Cell 33: 389-396.

Funk JO and Kind P (1997). Cell cycle control, genetic instability and cancer. Hautarzt 48: 157-165.

Gurion R, Gafter-Gvili A, Vidal L, Leader A, et al. (2013). Has the time for first-line treatment with second generation tyrosine kinase inhibitors in patients with chronic myelogenous leukemia already come? Systematic review and meta-analysis. Haematologica 98: 95-102.

Hou X, Wang S, Zhou Y, Xu Z, et al. (2005). Cyclin D1 gene polymorphism and susceptibility to childhood acute lymphoblastic leukemia in a Chinese population. Int. J. Hematol. 82: 206-209.

Howe D and Lynas C (2001). The cyclin D1 alternative transcripts [a] and [b] are expressed in normal and malignant lymphocytes and their relative levels are influenced by the polymorphism at codon 241. Haematologica 86: 563-569.

Hu Z, Zhou Z, Xiong G, Wang Y, et al. (2014). Cyclin D1 G870A polymorphism and the risk of hepatocellular carcinoma in a Chinese population. Tumour Biol. 35: 5607-5612.

Hunter T and Pines J (1991). Cyclins and cancer. Cell 66: 1071-1074.

Hunter T and Pines J (1994). Cyclins and cancer. II: Cyclin D and CDK inhibitors come of age. Cell 79: 573-582.

Knudsen KE, Diehl JA, Haiman CA and Knudsen ES (2006). Cyclin D1: polymorphism, aberrant splicing and cancer risk. Oncogene 25: 1620-1628.

Li J, Luo F, Zhang H, Li L, et al. (2014). The CCND1 G870A polymorphism and susceptibility to bladder cancer. Tumour Biol. 35: 171-177.

Liu J, Liao Q, Zhang Y, Sun S, et al. (2012). Cyclin D1 G870A polymorphism and lung cancer risk: a meta-analysis. Tumour Biol. 33: 1467-1476.

Lu F, Gladden AB and Diehl JA (2003). An alternatively spliced cyclin D1 isoform, cyclin D1b, is a nuclear oncogene. Cancer Res. 63: 7056-7061.

Nissen NN and Martin P (2002). Hepatocellular carcinoma: the high-risk patient. J. Clin. Gastroenterol. 35: S79-S85.

Pakakasama S, Chen TT, Frawley W, Muller CY, et al. (2004). CCND1 polymorphism and age of onset of hepatoblastoma. Oncogene 23: 4789-4792.

Sacks HS, Berrier J, Reitman D, Ancona-Berk VA, et al. (1987). Meta-analyses of randomized controlled trials. N. Engl. J. Med. 316: 450-455.

Sameer AS, Parray FQ, Dar MA, Nissar S, et al. (2013). Cyclin D1 G870A polymorphism and risk of colorectal cancer: a case control study. Mol. Med. Rep. 7: 811-815.

Solomon DA, Wang Y, Fox SR, Lambeck TC, et al. (2003). Cyclin D1 splice variants. Differential effects on localization, RB phosphorylation, and cellular transformation. J. Biol. Chem. 278: 30339-30347.

Wang J, Zhan P, Chen B, Zhou R, et al. (2010). MTHFR C677T polymorphisms and childhood acute lymphoblastic leukemia: a meta-analysis. Leuk. Res. 34: 1596-1600.

Zeybek U, Yaylim I, Ozkan NE, Korkmaz G, et al. (2013). Cyclin D1 gene G870A variants and primary brain tumors. Asian Pac. J. Cancer Prev. 14: 4101-4106.

Zhang YJ, Chen SY, Chen CJ and Santella RM (2002). Polymorphisms in cyclin D1 gene and hepatocellular carcinoma. Mol. Carcinog. 33: 125-129.

Zhuo W, Zhang L, Wang Y, Zhu B, et al. (2012). Cyclin D1 G870A polymorphism is a risk factor for esophageal cancer among Asians. Cancer Invest. 30: 630-636. 